\title{
Electronic Service Delivery in the Public Sector: Understanding the Variance of Citizens' Resistance
}

\author{
Martin Barth \\ University of Mannheim \\ Martin.Barth@bwl.uni-mannheim.de
}

\author{
Daniel Veit \\ University of Mannheim \\ Veit@bwl.uni-mannheim.de
}

\begin{abstract}
After the private sector the public sector also tries to benefit from the advantages of electronic service delivery, in particular from lower costs and higher accessibility. While more and more services are available electronically, citizens' usage rates lag behind. The e-government research community identified this issue and increasingly analyzes the demand side: Researchers investigated the acceptance of existing electronic services (e.g. implemented taxpaying systems) or means to reach "more resistant" groups of society by electronic services, e.g. citizens without internet access. Besides more resistant users, which are harder to convince of electronic services, there might be more demanding types of services. Such services are, from the perspective of almost all users, less amenable for electronic delivery than other (i.e. not demanding) services.

This study employs qualitative research methods in a case study design and identifies three main (service related) determinants that cause citizens to prefer traditional, i.e. physical and personal contact, over impersonal "virtual" contact.
\end{abstract}

\section{Introduction}

In accordance with Overby a virtual process can be defined as "a process in which physical interaction between people and/or objects has been removed" [30, p. 278]. In contrast a physical process still involves such physical interactions. E-commerce has virtualized shopping processes, which had previously been conducted physically. Other examples are banking processes, which can be performed in a virtual manner as online banking. Furthermore, project meetings are virtualized by use of video conferencing, or friendship development processes via social networks and virtual worlds.
Also in the public sector countries and their public authorities are investing significant amounts in egovernment to enable virtual service delivery by electronic services. For example, the US federal government is expected to have spent more than 7 billion US dollars on electronic services in 2009 [3, p. 27]. Governments are attracted by the potential of electronic service delivery to improve service quality and reduce costs $[22$, p. 324,38, p. 1], which is of particular importance in deeply indebted countries. Thus, the number of electronic services is continuously growing also in Europe. For the European Union (EU) the statistical office reports that the online availability of 20 selected public services has grown from $41 \%$ in 2004 to $62 \%$ in 2007 [15]. Unfortunately, in e-government citizens' usage rates lags behind. In 2009 only $29 \%$ of the European Union's individuals used some e-government service (including pure informational services such as reading a public website), this is only slightly more than in 2005 with $23 \%$ [16]. Transactional electronic services (i.e. returned online forms) were only conducted by $12 \%$ of EU's citizens in 2009 [17].

Previously focused on analyzing the provision of electronic services in countries and municipalities (i.e. supply-side), e-government research has identified this challenge and is now increasingly tackling the demand side: The acceptance of existing electronic services (e.g. implemented taxpaying systems) and means to reach "more resistant" groups of society have been studied: e.g. citizens without internet access ("first level digital divide") or elderly people without the required skills ("second level of digital divide") [37, p. 7].

Besides more resistant users, which are harder to convince of electronic services, there might be more demanding types of services. In the private sector, for instance, electronic shopping of clothes is far less popular than electronic shopping of books. For both product groups, elderly people may buy less than their fair share, but the digital divide does not explain 
why, within each user group, e-commerce for books is more popular than for clothes. In the same manner demanding public services would be, from the perspective of almost all citizen groups, just less amenable for electronic delivery than other services. Thus, public service providers should focus on the implementation of other services first and know the key challenges for each of these services to be able to address them accurately at a later stage. Hence, the identification of, from the citizens' perspective, more demanding services should be possible (a) before the costly implementation of the electronic services and (b) just based on service characteristics, not the specific implementation of the service. This task will become even more essential in the future, since after pure informational and other "low hanging fruit" services, the more complex transactional services will have to be investigated for electronic service delivery $[2,25]$. For these transactional services the selection becomes far more challenging, since, beside the implementation effort, the range of potential resistance roots increases.

Providing guidance for public institutions requires theoretical knowledge and a deep understanding of the demand side. Therefore the research in this study addresses this challenge by focusing on the following two research questions:

Do citizens prefer traditional service delivery for some services, but virtual services delivery for others? And if so why?

Which characteristics of a public process affect citizens' resistance to conduct it virtually?

These research questions are addressed by interviewing citizens regarding their preference for virtual or physical service delivery for selected public processes in a multiple case study design.

\section{Theoretical foundation}

Due to space restrictions only a short extract of the related work and theoretical foundation can be given. The following sections focus on the theoretical foundations having a direct influence on the conceptual model and its propositions.

\subsection{Process virtualization theory}

Electronic service delivery differs from traditional face-to-face service delivery by the absence of physical interaction between the citizen, the public sector employees and physical objects or documents. Overby [30] names the underlying process of such impersonal services a "virtual process" and identifies "process virtualizability" as a critical prerequisite for a successful, widely accepted electronic service. Processes less amenable for virtualization might still be virtualized but require far more effort and innovation to be successful.

In most cases the individuals decide, if they prefer to perform a process in the traditional physical or in a virtual manner. For this reason Overby analyzes "virtualizability" of a process from the individual's not the provider's perspective and defines the influence factors accordingly. Hence, in the sense of process virtualization theory, a process more amenable to virtualization will be conducted in a virtual manner by its target group without significant resistance. In contrast, the targeted individuals will not naturally perform a process virtually, which is less amenable for virtualization. Overby introduces four factors all affecting virtualizability of a process negatively:

"Sensory requirements" are defined as "the need for process participants to be able to enjoy a full sensory experience of the process and the other process participants and objects. Sensory experience include seeing, hearing, smelling, touching, and tasting [...]" $[30$, p. 280]. For instance, if the participant likes to smell an object, e.g. perfume, within the process, this has a negative influence on its "virtualizability".

The second construct, "relationship requirements", is defined as "the need for process participants to interact with one another in a social or professional context. Such interaction often leads to knowledge acquisition, trust development, and friendship development" [30, p. 281]. For example, if the participants of a process wish a long-term and personal relationship between each other, e.g. to reduce transaction costs, this has a negative influence on the "virtualizability" of this process.

"Synchronism requirements" are the third construct proposed to affect process virtualizability negatively. Overby [30, p. 281-282] defines it as "the degree to which the activities that make up a process need to occur quickly with minimal delay". Thus, if the participant of a process wants immediate results, e.g. direct handover of a physical product, this has a negative effect on the process "virtualizability".

"Identification and control requirements" are proposed as the fourth construct and defined as "the degree to which the process requires unique identification of process participants and the ability to exert control over/influence their behavior" [30, p. 282]. If a process requires the secure identification of the participants in a process, e.g. to make sure the contractual provisions are fulfilled, this has a negative influence on process "virtualizability".

The "process virtualizability", the dependent variable of the process virtualization theory, "can be 
measured either as: adoption of the virtual process, or the quality of the outcomes of the virtual process" [30, p. 279]. Hence, the high adoption rate, respectively the high value share, of e-commerce for buying books demonstrates high virtualizability of the book shopping process. Since the process virtualization theory is relatively new, it has not been applied or tested widely. Only Overby and Konsynski [31] presented a study providing empirical evidence for the theoretical model. An empirical analysis of Overby's model in the public sector is therefore highly interesting both from a theoretical and a practical perspective $[30,31]$.

\subsection{Relevant e-commerce literature}

The e-commerce research community agrees that "[s]ome product categories are more suitable for online shopping than other categories" [29, p. 113]. Product categories mostly listed in this context are traveling, tickets, books, electronics, music etc. [14, p. 325,20 , p. 706,23 , p. 456].

Despite this agreement, only limited consensus exists about the concrete product or service attributes that determine whether a product or service is amenable for the online channel or not. Mayo [28, p. 286] argues, "[...] the products [that] respondents will not buy online seem to have more similarities than products [which]consumers will buy online." Based on a literature review of Chang et al. [6] the relevant product and service attributes can be structured in three groups: Importance of product/service, ability to judge the quality of the product/service, and perceived product/service risk.

With regard to the first group, products and services, which are associated with high consumer involvement, are less amenable to online shopping than products and services without these attributes $[26,28]$. While the same was hypothesized for costly and less frequently consumed products/services the empirical results are ambiguous: Phau and Poon [33] found empirical evidence for this relation, but Vijayasarathy [42] was not able to show such an influence.

Second, the ability to judge the quality of the product/service affects its fit to the online channel. Tangible products as well as products and services with high complexity or inspection desire are less amenable to online shopping. For example, with respect to tangibility Phau and Poon [33, p. 103] argue: "One weakness of the Internet is that it can realistically reproduce only two of our five senses, namely, sight and sound. One cannot feel, smell or taste products that are advertised on the $W W W$. This limitation will restrict the kind of products that are sellable on the Internet." The empirical results for standardized products and services, i.e. with low differentiation, are contradicting: Even though Elliot and Fowell [14] identified a positive effect for standardized products and services on amenability to online shopping, Phau and Poon [33] found the inverse influence.

"Perceived product/service risk" constitutes a third definite factor emphasized in three empirical studies [18-20]. It "is associated with the product itself" [4, p. 99] and distinct to risks related to the sales channel or the implementation of the website. Products and services with higher perceived risk are less amenable to e-commerce.

\subsection{Relevant information system (IS) theory}

Two research streams with relevance to this research project have been identified within the field of information systems: (1) the technology acceptance model (TAM) and its successors (e.g. TAM2, UTAUT) and (2) the innovation diffusion theory (IDT). Both found factors influencing the acceptance of concrete technology products or new innovations $[24,41]$ by individuals.

Unfortunately, the structural equation models of these theories only facilitate optimal implementation of already selected services (e.g. focus on ease of use) or optimal diffusion of already implemented services (e.g. social influence), but does not guide the selection process itself. The determinants of these theories are dependent on the type of implementation, these constructs can not be directly adapted to this project's research model: e.g. "effort expectancy" or the "user support" [41] depends on realization of the technology product and its support approach.

These IS theories have been widely applied in all kinds of technology acceptance and innovation diffusion scenarios, also in e-government [32, 39]. Interestingly the models were adjusted to the respective scenario at hand and updated with new constructs to the concrete context. Hence, an investigation of the types of adjustments and updates made when these models were transferred from the private to the public sector can be very helpful to arrange the transfer of other private sector models in this study in a similar manner.

To identify the most influential constructs used on top of the original technology acceptance and diffusion models in the field of e-government, a comprehensive literature review was conducted on 10 top ranked IS and public administration journals as well as on the most cited journal articles on this topic. Two closely related construct categories were frequently employed, namely perceived risk and trust 
[e.g. 7, 43]. In the context of this research project, characteristics of public sector processes, not characteristics of individuals, organizations, or technologies are examined. While for the latter trust is a well established construct (e.g. trust in government or trust in the internet), for characteristics of public sector processes the risk perception is more appropriate.

The perceived risk of a process can be defined as the risk associated with conducting the process.

Perceived risk can not only be differentiated based on its investigation object, e.g. process or technology, but also on its origin. Featherman and Pavlou [18] defined based on the seminal work of Bauer [1] seven facets of perceived risks. In e-government and for the perceived risk of processes not all of these facets are equally important. According to the results of the literature review the facets performance risk and privacy risk are most frequently used in this context. As Horst et al. [21, p. 1839] argue, the perceived risk influence might become even more important in the future after diffusion of fully transactional services, since "[t]he risks of electronic services on the transactional level are more profound than at the informational level."

\subsection{Theory from communication research}

Media richness theory is based on the idea that media differs in richness [8-10]. Consequently, depending on the type of communication task the medium with the adequate richness has to be chosen for optimal effectiveness.

The theory defines richness as the capacity of providing immediate feedback, language variety, personalization and multiple channels or cues. Hence, the theory sees face-to-face contact as the richest medium, followed by telephone contact, e-mails and websites [10, 35, 36]. Draft and Lengel, the originating authors of media richness theory, discussed several factors for distinguishing communication tasks and accordingly choosing the right medium [11, p. 259]. Initially they described equivocality and uncertainty of communication tasks as the relevant differentiation factors [10, p. 357], but in later publications uncertainty was omitted and three other factors were used interchangeably with equivocality: routineness, emotional content and complexity [11, p. 259].

Independent of the factors used the core idea stays the same: The higher the equivocality of a task, the higher should be the richness of the selected medium. For less equivocal or complex tasks a less rich medium is optimal, since rich media, such as face-to- face contact, is typically more time-consuming or expensive.

While there are only a few studies applying media richness theory in the field of e-government, they come to very similar results [13, p. 190-191]. These publications transfer the theory from its pure communication origin, i.e. how to communicate, to a 'transaction-channel' selection, i.e. channel selection for transactional public services beyond pure communication tasks. The work in this field, with its origin in public sector channel management, relates closely to our research questions. The researchers propose task complexity and task ambiguity as the relevant factors for distinction between public services that require rich versus less rich media [13, $34,35,40]$. Besides these two (media-richness) constructs no further task or process related constructs are proposed and tested in their models.

\section{Conceptual model}

Based on the theoretical foundation discussed above nine propositions are hypothesized using nine different independent variables (see Figure 1). All independent variables are assumed to have a (direct or indirect) positive influence on the dependent variable resistance towards conducting the (respective) public process virtually. The dependent variable has been changed with regard to the original publications of Overby [30, 31] to illustrate its independence from the implementation of the process and to highlight the models main purpose to identify specially demanding processes as well as its key resistance determinants.

The first three propositions are taken from the process virtualization theory [30] and defined accordingly (see subsection 2.1). To facilitate the understanding of the propositions the three independent variables transferred are slightly renamed.

Proposition 1: The greater the perceived Sensory Requirements (SR) of a public process, the higher is the citizens' resistance towards conducting this process virtually.

Proposition 2: The greater the perceived Relationship Requirement (RR) of a public process, the higher is the citizens' resistance towards conducting this process virtually.

Proposition 3: The greater the perceived Immediate Results Requirements (IRR) of a public process, the higher is the citizens' resistance towards conducting this process virtually.

The fourth independent variable of the process virtualization theory has been replaced by two new 
variables and propositions to incorporate the influence of the perceived risk associated with the public process. The relevance of this influence has been highlighted by the e-commerce literature, by the publications on differences between e-commerce and

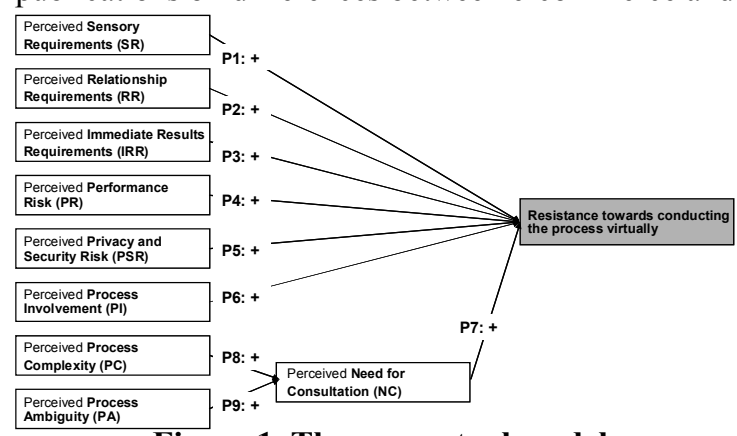

Figure 1. The conceptual model

e-government, and by the e-government papers based on TAM/UTAUT and IDT.

As discussed above the perceived risk in this field of e-government is made up of mainly two risk facets, namely performance risk and privacy/security risk. "Perceived performance risk refers to the possibility that the [... public process/service] will not function as expected and/or will not provide the desired benefit [19, p. 145]." Perceived privacy and security risk is defined as the "[p]otential loss of control over personal information, such as when information about you is used without your knowledge or permission. The extreme case is where a consumer is 'spoofed' meaning a criminal uses their identity to perform fraudulent transactions" [18, p. 455].

Proposition 4: The greater the perceived Performance Risk (PR) of a public process, the higher is the citizens' resistance towards conducting this process virtually.

Proposition 5: The greater the perceived Privacy and Security Risk (PSR) of a public process, the higher is the citizens' resistance towards conducting this process virtually.

In accordance with the e-commerce literature the proposition 6 is formulated as follows:

Proposition 6: The greater the perceived Process Involvement (PI) of a public process, the higher is the citizens' resistance towards conducting this process virtually.

Perceived process involvement is defined as " $[a]$ person's perceived relevance of the [... public process] based on inherent needs, values, and interests" [45, p. 342].

Overby $[30, \quad$ p. 280] subsumed individual's willingness to use his or her senses and "the overall sensation that participants feel when engaging in the process" in the same variable "sensory requirements". The latter aspect is similar to process involvement (barrier), but since these two influences are of very different nature, two different variables are introduced for these in this conceptual model (see propositions 1 and 6).

The last three propositions rely on the e-government literature based on media richness theory: According to these publications citizens prefer personal consultation for complex or ambiguous tasks and therefore visit the respective public agency.

Proposition 7: The greater the perceived Need for Consultation (NC) of a public process, the higher is the citizens' resistance towards conducting this process virtually.

Process "complexity is about the number of interrelated actions a citizen has to take in order to solve one's problem. The more interrelated actions the more complex a [... public process] becomes. The higher [... process] complexity becomes, the more information an individual has to process in order $[\ldots]$ " to conduct the process correctly $[13$, p. $194-$ 195].

Proposition 8: The greater the perceived Process Complexity (PC) of a public process, the higher is the perceived need for consultation.

Process "ambiguity is about not feeling sure of how to interpret information telling what to do. To remove or reduce problem ambiguity government needs to confirm whether or not a citizen's interpretation is correct" [13, p. 194].

Proposition 9: The greater the perceived Process Ambiguity (PA) of a public process, the higher is the perceived need for consultation.

\section{Research methodology}

To analyze the conceptual model described above, an explanatory multiple case study approach was chosen. In accordance with Yin [44], this approach seemed most appropriate to answer our main research questions on whether and why citizens are resistant to conduct some public processes virtually, while they prefer such service delivery for other processes. The qualitative approach, in contrast to a quantitative survey design, also left room for extensions of the conceptual model in an exploratory manner through identification of missing constructs or underlying issues and themes.

\subsection{Case study design}

To ensure rigorous research the case study research approach by Yin [44] was closely followed. One major distinction of the case study method to other popular research methods such as empirical survey 
design lies in the approach employed for generalization. In the latter, so called "statistical generalization"[44], an inference is made based on the statistical analysis of a representative sample for the whole population. Due to typically small numbers of cases statistical generalization is not possible [44]. In case studies analytical generalizability is achieved by combining interesting cases to disqualify alternative explanations in the so called "replication logic"[44]. Having multiple cases boosts the analytical potential of the research project, since only in this condition cross-case comparisons are feasible. For these reasons, in this research project a multiple case study design is followed: Each case study investigates (citizens' resistance for) one (potentially virtualized) public service. In Germany, a federally organized country, three hierarchical levels for public service delivery can be distinguished: the federal government, its 16 federal states and the roughly 12,000 municipalities $[27$, p. 571]. Since municipalities deliver the major share of public services to its respective citizens, this level seemed particularly appropriate for our analysis. Hence, in cooperation with a midsize German city, with roughly 300,000 citizens, five of its public processes were selected.

The selection was based on three independent expert interviews with department managers, responsible for public service delivery to citizens. They were asked to recommend services, which (a) are transactional in nature, (b) are requested several times a day, (c) still require physical appearance at the municipality and (d) which are assessed by the managers as either clearly amenable or highly inappropriate for virtual service delivery from the citizens' perspective. The following five public processes were chosen: (1) PERsonal registration (PER): E.g. after moving to a new address the Germans need to register their new address in the municipality they are living in. (2) PASsport application (PAS): Germans do apply for a new passport in their local municipality, even though the passports are produced centrally at the federal level. (3) VEHicle Registration (VEH): After buying a new car or moving with a car to a new city, Germans need to register their vehicle at the municipality they are living in, to get their license plate. (4) CHUrch deregistration (CHU): In Germany the state is responsible to collect the so called church tax, which is then forwarded to the respective church. For this reason the municipalities have a register of the affiliation of their citizens with churches. By deregistration in the municipality the citizen can save this tax and thereby is no longer a member of the respective church. (5) CIVil marriage (CIV): The civil marriage consists of two steps, which are both conducted by the municipalities: The registration for civil marriage and the civil wedding ceremony. Since in the expert interviews from the five chosen processes two were estimated as being amenable and three as being inappropriate from the citizens' perspective, "literal replication", i.e. comparison of multiple similar cases, as well as "theoretical replication", i.e. comparison of cases with contrasting results for theoretically grounded reasons, was targeted for generalizablity [12, 44].

An interview protocol was designed to guide the interviews with citizens requesting the respective service at the municipality. The protocol included the semi-structured, open-ended questions to ensure reliability and consistency for cross-case comparison, but left room for reaction on the interviewee's responses.

Four pilot interviews were conducted and analyzed before the data collection phase. Based on the interview transcripts the interview protocol was slightly refined to improve convenience and intelligibility.

\subsection{Sampling and data collection procedure}

Between April and June 2010 for each of the five processes 5-7 interviews were conducted in person with citizens that requested the respective service in the municipality. On average an interview took roughly 20 minutes. On each interview day the interview period started right when the municipality opened and ended when it was closed. The interviewer randomly selected the next citizen for the next interview by picking the citizen leaving the office after the most recent interview had been finished. All citizens identified with this approach were interviewed, if they had requested one of the selected services and agreed to participate. The interviewer guaranteed privacy and confidentiality to all interviewees to ensure true and open responses. Each citizen was only interviewed once and about one service only. The interviews were audio taped and transcribed afterwards. Only one interviewee declined the permission to record the interview and thus in this case notes were taken during the interview.

\subsection{Data analysis}

The transcripts of the 28 interviews were aggregated and stored in the case study database, which comprised 27,963 words and 85 pages of text. The 
data analysis was conducted using the software ATLAS.ti ${ }^{1}$ and structured in three steps.

First, the coding scheme was developed based on the recommendations of Boyatzis by the first author, who is also the first coder [5]. The coding scheme was developed "theory-driven" [5] and consisted of one code for each proposition of the conceptual model. Only for the proposition process involvement, which lead the interviewees to the comparison of multiple processes, a second code was introduced. This ensured that differences between the perception of the process at hand and an abstract statement concerning other processes could be captured. Two codes were used to identify the intent to use the physical process or intent to use the virtual process respectively. These two codes were necessary to make sure that exactly one option (virtual or physical process), but not zero or both options were preferred by the interviewees throughout the interview. Resistance towards conducting the process virtually (i.e. the dependent variable) was identified, if for one interview transcript the intent to use the physical process was coded, but not the second (control) code. Finally, High Effort to Visit administration (HEV) was coded as a "control code", if the interviewee stated the effort required to come to the municipality as high.

After initial discussions with the second coder, a research colleague, the coding scheme was refined for intelligibleness. Both coders had previous experience with e-government research and interview coding.

Second, both coders coded 4 interview transcripts form the pre-test independently from each other based on the previously defined coding scheme. Based on the differences in the coding results, the coders refined the coding scheme further, to guarantee a common understanding of all codes.

In the third step, both coders coded the 28 interview transcripts independently from each other, strictly on basis of the finalized coding scheme. The inter-rater reliability, calculated as the percentage of agreement on presence [5, p. 154-155], ranges from 77 to $100 \%$ with an average of $88 \% .100 \%$ agreement was given on the codes identifying the intent to use either the physical or the virtual process. This is no surprise, since this question was asked very explicitly by the interviewer and was answered quite unambiguously by the participants. The final coding matrix contains the coding of the first coder, who had most experience with the content since he also conducted and transcribed the interviews.

\footnotetext{
${ }^{1}$ Vers. 6.1.13, http://atlasti.com, access date 1/06/2010
}

\subsection{Results}

All interviewees were asked if they would prefer to come to the municipality again to conduct the respective requested service given a new virtual service delivery opportunity from home would be provided as an alternative in future. As shown in Table 1, based on the coded results for "intent to use the virtual process" two groups can be distinguished: While for civil marriage and vehicle registration the vast majority, $75-100 \%$, of interviewees are resistant to use the virtual service, most interviewees requesting the other three public services would prefer the virtual service, resistance rates range from $20 \%$ to $33 \%$. Interestingly, this difference between the processes is not caused by an unequal share of persons not having access to the internet or not capable of conducting a public process via internet, since the same trend exists for the sub-group of interviewees not having any of these issues (see Table 1). To exclude this potential bias the following analysis focuses on the interviewees, who stated not to have any of the described digital divide restrictions.

4.4.1. Civil marriage (CIV). The results of the code analysis investigating the (process specific) resistance factors for virtual service delivery for the civil marriage process are shown in Table 2. The number of interviewees mentioning the code and the respective relative share are listed. Bold figures indicate the main determinants that are mentioned by more than half of the interviewees. Three main determinants could be identified, mentioned by 80 100\%: Process Involvement (PI) is the strongest determinant for the resistance regarding the virtual process:

Table 1. Two demanding public processes

\begin{tabular}{|l|l|l|l|l|}
\hline $\begin{array}{l}\text { Public } \\
\text { process }\end{array}$ & $\begin{array}{l}\text { \# of } \\
\text { inter- } \\
\text { views }\end{array}$ & $\begin{array}{l}\text { Thereof } \\
\text { with intent } \\
\text { to use } \\
\text { physical } \\
\text { process } \\
\text { in \% (abs.) }\end{array}$ & $\begin{array}{l}\text { \# of } \\
\text { inter- } \\
\text { viewees } \\
\text { without } \\
\text { access } \\
\text { or skill } \\
\text { restric- } \\
\text { tion }\end{array}$ & $\begin{array}{l}\text { Thereof } \\
\text { with intent } \\
\text { to use } \\
\text { physical } \\
\text { process } \\
\text { in \% (abs.) }\end{array}$ \\
\hline $\mathrm{CIV}^{2}$ & $7^{3}$ & $\mathbf{1 0 0 \% ( 7 )}$ & 5 & $\mathbf{5}$ \\
\hline $\mathrm{VEH}^{2}$ & $6^{3}$ & $\mathbf{8 3 \%}(5)$ & 4 & $\mathbf{1 0 0 \% ( 5 )}$ \\
\hline $\mathrm{PER}^{2}$ & 5 & $20 \%(1)$ & 5 & $20 \%(1)$ \\
\hline $\mathrm{PAS}^{2}$ & 5 & $20 \%(1)$ & 4 & $25 \%(1)$ \\
\hline $\mathrm{CHU}^{2}$ & 5 & $20 \%(1)$ & 3 & $33 \%(1)$ \\
\hline
\end{tabular}

\footnotetext{
${ }^{2}$ See section 4.1 for full names and explanations of the processes

${ }^{3}$ Additional interviews were conducted for CIV and VEH to have at least 4 interviews per process in the subgroup with access and skills.
} 
"There are some things, which are personally important. Civil marriage is one of these. I would also not like to deliver my baby with a remote connection to my mid-wife." (P16:61)

Immediate Results Requirements (IRR) and Sensory Requirements (SR) are the second strongest determinants:

"[In the virtual process] I have to write e-mails and then I have to wait for the reply. Here, I get the reply right away." (P28:13)

"Even a videoconference system would be a restriction. This would not be good enough for me." (P24:34)

4.4.2. Vehicle registration (VEH). The results of the code analysis investigating the (process specific) resistance factors for virtual service delivery for the vehicle registration process are shown in Table 2 analogously to the civil marriage process. The same three main determinants identified for civil marriage are also discovered for vehicle registration. In addition, two more main determinants are detected. Immediate Results Requirements (IRR) is the strongest determinant:

"Yes, it is urgent. I bought the car yesterday and promised the seller to register it under my name today. For this reason it has to be completed today." (P27:37)

"I would like to finish it right away, to tick it off my to-do list. This is important to me." (P8:36)

Process Involvement (PI), Sensory Requirements (SR), Performance Risk (PR) and, Privacy and Security Risk (PSR) are identified as the second strongest determinants:
"Yes, this [vehicle registration] is important to me.[...] the involvement is relevant. Unimportant things I would do at home, but things that are personally important to me I would only do on site." (P13:45)

"The personal contact is more important: talking to the official and seeing him in front of me." (P8:20)

"The main thing is, seeing that the paperwork and the [vehicle] registration work out fine." (27:24)

While the determinant privacy and security risk is mentioned frequently as barrier for the virtual vehicle registration process, most interviewees describe it as one of several determinants with limited influence on the decision process. This is also true for most of the occurrences of this code with regard to other processes.

"I do have some concerns [regarding the privacy and security]. These are no major concerns, rather miner ones." (P8:39)

In contrast to the majority, one interviewee would prefer the virtual process for vehicle registration. In this transcript none of the three main determinants, detected for the civil marriage process and for the resistant majority of interviewees questioned on the vehicle registration process, could be found. The dominant theme, mentioned by the interviewee multiple times on different questions, is saving time:

"During this time you can do other things. Time is money." (P14:14)

"The main thing is you save time to do other things." (P14:18)

The chance of saving time seems to eliminate or dominate the perception of any potential barrier for

Table 2. Coded determinants for demanding processes (as mentioned by resistant interviewees)

\begin{tabular}{|l|l|l|l|l|l|l|l|l|l|l|}
\hline Public process & $\mathbf{P I}^{4}$ & $\mathbf{I R R}^{4}$ & $\mathbf{S R}^{4}$ & $\mathbf{P S R}^{4}$ & $\mathbf{N C}^{4}$ & $\mathbf{P A}^{4}$ & $\mathbf{P R}^{4}$ & $\mathbf{R R}^{4}$ & $\mathbf{H E V}^{4}$ & $\mathbf{P C}^{4}$ \\
\hline Civil marriage \# of interviewees & $\mathbf{1 0 0 \%}$ & $\mathbf{8 0 \%}$ & $\mathbf{8 0 \%}$ & $40 \%$ & $40 \%$ & $40 \%$ & $20 \%$ & $20 \%$ & $20 \%$ & $0 \%$ \\
mentioned determinant in \% (abs) & $(5)$ & $(4)$ & $(4)$ & $(2)$ & $(2)$ & $(2)$ & $(1)$ & $(1)$ & $(1)$ & $(0)$ \\
\hline Vehicle registration \# of interviewees & $\mathbf{6 7 \%}$ & $\mathbf{1 0 0} \%$ & $\mathbf{6 7 \%}$ & $\mathbf{6 7 \%}$ & $0 \%$ & $0 \%$ & $\mathbf{6 7 \%}$ & $0 \%$ & $33 \%$ & $0 \%$ \\
mentioned determinant in \% (abs) & $(2)$ & $(3)$ & $(2)$ & $(2)$ & $(0)$ & $(0)$ & $(2)$ & $(0)$ & $(1)$ & $(0)$ \\
\hline
\end{tabular}

Table 3. Coded determinants for not-demanding processes (as mentioned by not resistant interviewees)

\begin{tabular}{|l|l|l|l|l|l|l|l|l|l|l|}
\hline Public process & $\mathbf{P I}^{4}$ & $\mathbf{I R R}^{4}$ & $\mathbf{S R}^{4}$ & $\mathbf{P S R}^{4}$ & $\mathbf{N C}^{4}$ & $\mathbf{P A}^{4}$ & $\mathbf{P R}^{4}$ & $\mathbf{R R}^{4}$ & $\mathbf{H E V}$ & $\mathbf{P C}^{4}$ \\
\hline Personal registration \# of interviewees & $0 \%$ & $25 \%$ & $0 \%$ & $25 \%$ & $50 \%$ & $25 \%$ & $0 \%$ & $0 \%$ & $25 \%$ & $0 \%$ \\
mentioned determinant in \% (abs) & $(0)$ & $(1)$ & $(0)$ & $(1)$ & $(2)$ & $(1)$ & $(0)$ & $(0)$ & $(1)$ & $(0)$ \\
\hline Passport application \# of interviewees & $0 \%$ & $0 \%$ & $0 \%$ & $0 \%$ & $0 \%$ & $0 \%$ & $\mathbf{6 7 \%}$ & $0 \%$ & $33 \%$ & $0 \%$ \\
mentioned determinant in \% (abs) & $(0)$ & $(0)$ & $(0)$ & $(0)$ & $(0)$ & $(0)$ & $(2)$ & $(0)$ & $(1)$ & $(0)$ \\
\hline Church deregistration \# of interviewees & $0 \%$ & $0 \%$ & $0 \%$ & $50 \%$ & $0 \%$ & $0 \%$ & $0 \%$ & $0 \%$ & $50 \%$ & $0 \%$ \\
mentioned determinant in \% (abs) & $(0)$ & $(0)$ & $(0)$ & $(1)$ & $(0)$ & $(0)$ & $(0)$ & $(0)$ & $(1)$ & $(0)$ \\
\hline
\end{tabular}

Table 4. Coded determinants for not-demanding processes (as mentioned by outlier, i.e. resistant interviewees)

\begin{tabular}{|l|l|l|l|l|l|l|l|l|l|l|}
\hline Public process & $\mathbf{P I}^{4}$ & $\mathbf{I R R}^{\mathbf{4}}$ & $\mathbf{S R}^{\mathbf{4}}$ & $\mathbf{P S R}$ & $\mathbf{N} \mathbf{C}^{\mathbf{4}}$ & $\mathbf{P} \mathbf{A}^{\mathbf{4}}$ & $\mathbf{P R}$ & $\mathbf{R} \mathbf{R}^{\mathbf{4}}$ & $\mathbf{H E V}$ & $\mathbf{P} \mathbf{C}^{\mathbf{4}}$ \\
\hline Personal registration total occurrences & 0 & $\mathbf{1}$ & 0 & 0 & 1 & 1 & $\mathbf{3}$ & 0 & 0 & 0 \\
\hline Passport application total occurrences & 0 & $\mathbf{1}$ & $\mathbf{3}$ & 0 & 0 & 0 & 0 & 0 & 0 & 0 \\
\hline Church deregistration total occurrences & 0 & 0 & $\mathbf{2}$ & 1 & 1 & 1 & 0 & 1 & 0 & 0 \\
\hline
\end{tabular}

\footnotetext{
4 See Figure 1 for the full names of the determinants
} 
using the virtual process for this interviewee. This assumption is further supported by the fact that the seldom identified code High Effort required to Visit the administration (HEV) is clearly found in this transcript.

4.4.3. Personal registration (PER) in municipality, Passport application (PAS) and Church deregistration (CHU). The results of the code analysis investigating the (process specific) determinants for virtual service delivery for the three remaining processes, for which respective interviewees showed no resistance, are shown in Table 3 analogously. Only for the passport application process one code was identified in more than $50 \%$ of the interview transcripts, namely Performance Risk (PR):

"Yes, [in the virtual process] something can go wrong. [...] This is a barrier." (P11:36)

Although this determinant was also detected as one barrier for the vehicle registration process, none of the three main determinants identified for both demanding services, namely civil marriage and vehicle registration, were found as main determinants for one of the three remaining services, that were mainly perceived as amenable for virtualization:

"I think this [church deregistration] is one of many services and if you think twice you only have to do it once in your lifetime." (P21:59)

"If the processing [of personal registration] is delayed by multiple weeks, this would be a problem. But if it is done on the next day, for instance, this would not be an issue at all." (P1:22)

"If the user interface [of the passport application process] is self-evident, I will have no problem at all not talking to a real person. " (P3:20)

As shown in Table 1, for each of the three service one outlier was detected, i.e. one interviewee preferring the physical channel in contrast to all other interviewees questioned about the respective service. In each of these interview transcripts one of the three main determinants detected for both civil marriage and vehicle registration was identified. Furthermore, each of the interviews contained one different dominant theme, which was mentioned and coded multiple times in the respective interview (see Table 4). Multiple occurrences of the risk related codes performance risk and privacy and security risk, can be seen as indicator for a strong underlying individual characteristic like (low) risk tolerance.

\section{Discussion}

While no statistical generalization is possible based on case study data, the results provide clear support for three of the seven direct determinants proposed in the conceptual model: process involvement, immediate results requirements, and sensory requirements. These were identified as main determinants for citizens' resistance to conduct civil marriage and vehicle registration virtually. At the same time the determinants were not deemed important in the other processes, which are mainly perceived as amenable for virtualization from the citizens' perspective. Support was found for two additional propositions, namely privacy and security risk and performance risk. These risk oriented factors, were perceived additionally as main determinants to use a virtual vehicle process, but not for a civil marriage process. In particular the performance risk needs further support, since it has been found as barrier in the passport application process as well, which the interviewees mainly perceived as amenable for virtualization. Interestingly, no support has been identified for the two proposed determinants, need for consultation and relationship requirements. While the first is at least mentioned multiple times for the civil marriage and the personal registration process, the latter is barely named as barrier at all. Multiple interviewees motivate this attitude, by the low frequency of contacts with the respective municipality official. Hence, the behavior might be different for services, which are required more frequently or require more frequent contacts to the same officials.

By employing theoretical replication, new insights have been found that extent and advance the proposed conceptual model. Prior to this case study the conceptual model, did not include any variables based on individual characteristics for good reason. The model focuses on identification of challenging public processes for virtualization, not on predicting the behavior of each single individual. But the analysis and comparison of the few outliers, i.e. interviewees that preferred the other option in contrast to the vast majority of the interviewees questioned about the respective process, provides some initial support for moderators influencing the perception and the magnitude of the main determinants based on individual characteristics, in addition to typical digital divide indicators. In particular risk tolerance and permanent lack of time, seems worth to be investigated as potential moderator of the main determinants in future. 


\section{Study limitations and future research}

The findings discussed above are based on a multiple, but single-site case study in two departments of a mid-size municipality. Thus, the findings may be influenced to a certain extent by the atmosphere, culture and working style of this organization. Furthermore the chosen public processes do not cover the full spectrum of public processes in a representative manner, in particular not the ones offered by public institutions other than municipalities. Consequently, the authors encourage research testing the findings on multiple sites, on a fuller spectrum of public services and on a large scale. Due to the data based on German citizens and the investigation of partly typical German public processes, the authors can not claim a general generalizability to other countries. But since the same problems of selecting the right public services for virtualization exists in many countries a thoughtful transfer to other countries and cultures is encouraged. Given the typical limitations of a qualitative research design with regard to statistical generalization, in particular reasonable number of cases and locations, the authors plan to expand this qualitative work by a representative, large-scale survey targeting German citizens.

\section{Conclusion}

The results of the multiple case study clearly reinforce the underlining hypothesis; citizens resistance to use virtual processes is influenced by the process type at hand. Distinct differences have been identified in the resistance of the five investigated public processes. The study also supports our practical motivation; municipality officials had serious difficulties, distinguishing public processes not yet offered virtually, as either amenable or resistant to virtualization from the citizens' perspective. For instance the officials expected citizens' resistance for a virtual passport application process in the expert interviews before the data collection phase, which was wrong according to the interviews. Also for the vehicle registration processes the officials were mainly unsure about the appropriate classification, before they knew the potential determinants from the conceptual model.

As discussed above support has been found for significant parts of the conceptual model, which explains the resistance of citizens regarding some but not all virtual public processes based on process characteristics. The three main determinants for resistance are immediate results requirements, sensory requirements and process involvement. Thus, two of the three propositions directly transferred from the process virtualization theory were supported. In contrast, for the third proposition coming from this theory, relationship requirements, no support has been found. The last main determinant, process involvement, originates in the e-commerce literature, which highlights the chances of transferring private sector knowledge to the e-government domain.

\section{References}

[1] Bauer, R., Consumer Behavior as Risk Taking, American Marketing Association, Chicago, 1960.

[2] Baum, C. and A. Di Maio, Gartner's four phases of egovernment model, Stamford, Gartner Group, 2000, pp. 15.

[3] Baumgarten, J. and M. Chui, E-government 2.0, McKinsey on Government, 2009, pp. 26-31.

[4] Bhatnagar, A., S. Misra and H. Rao, On risk, convenience, and Internet shopping behavior, Communications of the ACM, 2000, pp. 98-105.

[5] Boyatzis, R.E., Transforming Qualitative Information: Thematic Analysis and Code Development, SAGE Publications, Thousand Oaks, 1998.

[6] Chang, M., W. Cheung and V. Lai, Literature derived reference models for the adoption of online shopping, Information \& Management, 2005, pp. 543-559.

[7] Colesca, S. and L. Dobrica, Adoption and use of egovernment services: the case of Romania, Journal of Applied Research and Technology, Universidad Nacional Autonoma Mexico, 2008, pp. 204-217.

[8] Daft, R. and R. Lengel, Information richness: A new approach to managerial behavior and organizational design, JAI Press, Homewood, IL, 1984.

[9] Daft, R. and R. Lengel, Organizational information requirements, media richness and structural design, Management Science, 1986, pp. 554-571.

[10] Daft, R.L., R.H. Lengel and L.K. Trevino, Message equivocality, media selection, and manager performance: Implications for information systems, MIS Quarterly, 1987, pp. 355-366.

[11] Dennis, A. and S. Kinney, Testing media richness theory in the new media: The effects of cues, feedback, and task equivocality, Information Systems Research, 1998, pp. 256-274.

[12] Dubé, L. and G. Paré, Rigor in Information Systems Positivist Case Research: Current Practices, Trends, and Recommendations, MIS Quarterly, 2003, pp. 597-636.

[13] Ebbers, W., W. Pieterson and H. Noordman, Electronic government: Rethinking channel management strategies, Government Information Quarterly, 2008, pp. 181-201.

[14] Elliot, S. and S. Fowell, Expectations versus reality: a snapshot of consumer experiences with Internet retailing, International Journal of Information Management, 2000, pp. 323-336.

[15] Eurostat, E-government online availability Percentage of online availability of 20 basic public services, Eurostat, Luxembourg, 2008. 
[16] Eurostat, E-government usage by individuals by gender - Percentage of individuals aged 16 to 74 using the Internet for interaction with public authorities, Luxembourg, 2009.

[17] Eurostat, Individuals using the Internet for returning filled in forms to public authorities - Percentage of individuals aged 16 to 74, Luxembourg, 2009.

[18] Featherman, M.S. and P.A. Pavlou, Predicting eservices adoption: a perceived risk facets perspective, International Journal of Human-Computer Studies, 2003, pp. 451-474.

[19] Grewal, D., J. Gotlieb and H. Marmorstein, The moderating effects of message framing and source credibility on the price-perceived risk relationship, Journal of Consumer Research, 1994, pp. 145-153.

[20] Grewal, D., G. Iyer and M. Levy, Internet retailing: enablers, limiters and market consequences, Journal of Business Research, 2004, pp. 703-713.

[21] Horst, M., M. Kuttschreuter and J. Gutteling, Perceived usefulness, personal experiences, risk perception and trust as determinants of adoption of e-government services in The Netherlands, Computers in Human Behavior, 2007, pp. 1838-1852.

[22] Jaeger, P., The endless wire: e-government as global phenomenon, Government Information Quarterly, 2003, pp. 323-332.

[23] Jahng, J., H. Jain and K. Ramamurthy, Effective design of electronic commerce environments: a proposedtheory of congruence and an illustration, IEEE Transactions on Systems, Man and Cybernetics, Part A, 2000, pp. 456-471.

[24] Jeyaraj, A., J.W. Rottman and M.C. Lacity, A review of the predictors, linkages, and biases in IT innovation adoption research, Journal of Information Technology, 2006, pp. 1-23.

[25] Layne, K. and J. Lee, Developing fully functional Egovernment: A four stage model, Government Information Quarterly, 2001, pp. 122-136.

[26] Mathwick, C., N. Malhotra and E. Rigdon, The effect of dynamic retail experiences on experiential perceptions of value: an internet and catalog comparison, Journal of Retailing, 2002, pp. 51-60.

[27] Maurer, H., Allgemeines Verwaltungsrecht, Verlag C.H. Beck, München, 2009.

[28] Mayo, D., M. Helms and S. Inks, Consumer internet purchasing patterns: a congruence of product attributes and technology, International Journal of Internet Marketing and Advertising, 2006, pp. 271-298.

[29] Monsuwe, T., B. Dellaert and K. Ruyter, What drives consumers to shop online? A literature review, International Journal of Service Industry Management, 2004, pp. 102-121.

[30] Overby, E., Process virtualization theory and the impact of information technology, Organization Science, 2008, pp. 277-291.
[31] Overby, E. and B. Konsynski, Process Virtualization: A Theme and Theory for the Information Systems Discipline, SSRN, 2008.

[32] Patel, H. and D. Jacobson, Factors Influencing Citizen Adoption of E-Government: A Review and Critical Assessment, Proceedings of 16th European Conference on Information Systems, 2008.

[33] Phau, I. and S. Poon, Factors influencing the types of products and services purchased over the Internet, Internet Research: Electronic Networking Applications and Policy, 2000, pp. 102-113.

[34] Pieterson, W. and W. Ebbers, The use of service channels by citizens in the Netherlands: implications for multi-channel management, International Review of Administrative Sciences, 2008, pp. 95-110.

[35] Pieterson, W. and J. van Dijk, Channel Choice Determinants; An exploration of the factors that determine the choice of a service channel in citizen initiated contacts, Proceedings of 8th Annual International Digital Government Research Conference, 2007.

[36] Rice, R. and D. Shook, Relationships of job categories and organizational levels to use of communication channels, including electronic mail: A meta-analysis and extension, Journal of Management Studies, 1990, pp. 195229.

[37] Scholl, H., K. Barzilai-Nahon, J. Ahn, et al., ECommerce and e-Government: How Do They Compare? What Can They Learn From Each Other?, Proceedings of 42nd Hawaii International Conference on System Sciences, 2009.

[38] Teerling, M. and W. Pieterson, Government Multichannel Marketing: How to seduce citizens to the web channels?, Proceedings of 42nd Hawaii International Conference on System Sciences, 2009.

[39] Titah, R. and H. Barki, E-government adoption and acceptance: a literature review, International Journal of Electronic Government Research, 2006, pp. 23-57.

[40] van Deursen, A. and W. Pieterson, The Internet as a service channel in the Public Sector, Proceedings of International Communication Association Congress 2005, 2006.

[41] Venkatesh, V., M. Morris, G. Davis, et al., User acceptance of information technology: Toward a unified view, MIS Quarterly, 2003, pp. 425-478.

[42] Vijayasarathy, L., Product characteristics and Internet shopping intentions, Internet Research: Electronic Networking Applications and Policy, 2002, pp. 411-426.

[43] Warkentin, M., D. Gefen, P. Pavlou, et al., Encouraging citizen adoption of e-government by building trust, Electronic Markets, 2002, pp. 157-162.

[44] Yin, R., Case study research: Design and methods, Sage Publications, Thousand Oaks, California, 2008.

[45] Zaichkowsky, J., Measuring the involvement construct, Journal of Consumer Research, 1985, pp. 341352. 